\title{
Knowledge of HIV and HPV Infection, and Acceptance of HPV Vaccination in Spanish Female Sex Worker
}

\author{
C. Rodríguez-Cerdeira ${ }^{*}$, , E. Sánchez-Blanco ${ }^{2}$, A. Gutierrez ${ }^{2}$, A. Rodriguez-Rodriguez ${ }^{2}$ and \\ B. Sánchez-Blanco ${ }^{3}$
}

${ }^{I}$ Department of Dermatology, CHUVI/ University of Vigo, Vigo, Spain

${ }^{2}$ University of Vigo, Vigo, Spain

${ }^{3}$ Department of Emergency, CHUVY Vigo, Spain

\begin{abstract}
Objective: To examine the socioeconomic variables, lifestyles, and sexual behaviors of female sex workers; their knowledge about the risk of HIV and human papillomavirus (HPV) infection; the HPV vaccine, and their attitudes toward it.

Methods: 168 female sex workers (18-49 years old) filled out a questionnaire consisting of 5 parts with a total of 19 items.

Results: Knowledge of Pap smears was moderate; $47 \%$ of the participants had undergone at least one. Most respondents (52.4\%) had never heard of HPV. Most (88\%) recognized HIV as a virus that can be acquired sexually. For most women, recommendations from non governmental organizations (NGOs) (68.5\%) were the major influences in deciding to be vaccinated and learning how to take care of their health.

Conclusion: The cost-effectiveness ratios for HPV control and vaccination strategies would be more favorable if younger women are targeted and initial catch-up efforts are targeted to female sex workers by revising screening policies. Healthcare workers and volunteers in NGOs should be educated and trained about HIV, HPV infection, and HPV vaccination and its relationship with genital cancer.
\end{abstract}

Keywords: Female sex worker, HIV, HPV vaccination, sexual behavior.

\section{INTRODUCTION}

Prostitution (commercial sex work) is commonly regarded as "the oldest profession." The United Nations defines sex work as "the exchange of money or goods for sexual services, either regularly or occasionally, involving female, male, and transgender adults, young people, and children where the sex worker may or may not consciously define such activity as income-generating" [1]. The term covers a broad range of transactions, and the context in which these transactions occur has implications for assessing those at risk. While some may freely choose sex work as their occupation, others are coerced into it through violence, trafficking, or debt bondage [2].

The concept of commercial sex workers is rarely investigated, perhaps because of the often-informal nature of the workplace, associated stigma, and frequent illegality of the activity. We reviewed the literature on health, occupational risks, and safety among female sex workers (FSW) and identified cultural and local variations, and commonalities [3].

*Address correspondence to this author at the Servicio Dermatología. CHUVI. Hospital do Meixoeiro, 36200 Vigo. Spain;

Tel: 0034600536114; Fax: 0034986276416;

E-mails: carmen.rodriguez.cerdeira@sergas.es; crodcer@uvigo.es
In a comment in The Lancet, Groneberg et al. [4] mention the following occupational hazards that need to be taken into account in the lives of commercial sex workers: violence, harassment, infections, bladder problems, stress, depression, alcohol and drug addiction, latex allergy, and death. Legal regulations play extremely important roles in harm-reduction strategies. Alexander [5] adds musculoskeletal injuries to this list. Other occupational hazards include having money stolen by clients, being forced to have unprotected sex, and pregnancy [6].

Immigration status may further disempower sex workers. It is assumed that FSW who are migrants are at higher risk, although this may be due to the fact that some women are lured or sold across national borders specifically for the purposes of what is basically sexual slavery. Bautista et al. $[7,8]$ studied immigrant and non-immigrant FSW in Argentina and found that syphilis and hepatitis $\mathrm{C}$ were more common among Argentinean sex workers, whereas hepatitis $\mathrm{B}$ was more common among migrant FSW. Regression analysis indicates that the distinguishing factors among migrant FSW are being single, no occupation, and that lowpaid bar and cabaret workers are more likely to engage in anal sex.

Sex work involves specific interactions between the worker and client, and it is difficult to separate these 2 players in any attempt to improve and promote safe sex work 
encounters. Any attempt to demonize or justify the health and safety of one player over the other is not only philosophically challenging, but is likely to undermine the benefits of any effective health promotion approach in this industry, where risk to workers and clients is a dynamic interaction $[9,10]$. Recently, Langanke \& Ross [11] studied internet forums that serve to promote sexual safety and where clients of FSW share experiences; they highlight the potential importance of the internet as a medium not only as a marketplace for sexual services, but also for health and safety information for both clients and commercial sex workers [12].

There is evidence that effective interventions targeted at FSW involve health promotion approaches that are created by FSW themselves and their organizations. Furthermore, such approaches are the most effective and accepted by workers and clients [13-15].

Over the last few years, an increasing proportion of FSW in Spain are immigrants. This phenomenon is particularly prevalent in Northwestern Spain, where a very large proportion of FSW originate from other countries, the majority of which come from Latin America. It is likely that migrant FSW differ from others in terms of sociodemographic characteristics as well as their sexual and health-seeking behaviors. Although the HIV epidemic in Northwestern Spain has been driven predominantly by injection drug use, sexual transmission is playing an increasing role in the process. Findings revealed that FSW have knowledge about transmission of HIV infection and AIDS. However, they had misconceptions as to how HIV infection is transmitted, as they believed that poor nutrition and sharing facilities play a role. Knowledge of mechanisms of protecting themselves against infection, such as use of a condom during coitus was also evident [16]. On the other hand, screening programs in Spain are distributed unequally and have poor coverage. The target populations are heterogeneous and the criteria for their identification are unclear. Cytology laboratories frequently use their own terminology when issuing reports. It is difficult to determine the screening cost of cervical cancer in Spain, as no standardized procedure exists for cost analysis. Not all population censuses of tumors record in situ cervical carcinomas. The average screening coverage in Spain is $75 \%$, with large differences between communities. Around $35 \%$ of Spanish women have limited access to preventative follow-ups; this group is comprised of women older than 55 years, with low socioeconomic status, those living in rural areas, and FSW. According to this information, screening should be mixed. Women who do not attend health centers within an established time should be actively contacted. Thus, screening should commence at 25 years of age or within the first 3 years after starting sexual relationships. The diagnosis of high grade-cervical intraepithelial neoplasia (HG-CIN) should be incorporated into the population tumor records. Cost-benefit analysis programs should be initiated for the implemented screening programs $[17,18]$.

Awareness of sexually transmitted infections (STIs) and the virus that causes genital cancer is relatively low among FSW. In the present study, we surveyed FSW to examine their knowledge and behaviors regarding genital cancer screening and Pap smears, human papilloma virus (HPV), and the HPV vaccine, as well as their attitudes toward vaccination. We also examined their health status and its potential impact on the public health of the community in which they work.

\section{MATERIALS AND METHODS}

\section{Sample Size}

The estimated population of FSW in the provinces of Ourense and Pontevedra in Northwestern Spain is 1500, and the expected prevalence of HPV infection is $50 \%$; assuming a $95 \%$ confidence level and $7 \%$ error, it indicates that a sample of 168 women is required.

\section{Data Collection}

A descriptive, cross-sectional study was conducted on a sample of one hundred sixty-eight FSW aged 18-49 years involved in this project were recruited over the years 20102011 from different areas of prostitution (e.g., clubs, spas, the neighborhood, and the streets) in Pontevedra and Ourense through a mobile unit belonging to a related NGO and taken to health services, where they were interviewed and examined.

The participants who attended our clinic were asked to participate in the study. Their knowledge about cervical cancer screening, Pap smears, HIV, HPV, the HPV vaccine, and their attitudes toward vaccination were examined. An adhoc questionnaire (approximately 15 minutes long) was designed to collect information through face to face interviews conducted by a trained health worker in a confidential setting during or after the clinical examination. Participants signed an informed consent prior permission and the Ethics Committee of Galicia request.

The questionnaire consisted of 5 parts with a total of 19 items as follows:

1. Age (years)
a. $\quad 18-25$
b. $26-33$
c. $34-41$
d. $42-49$

2. Country
a. Spain
b. Eastern Europe
c. Latin America
d. Africa

3. Level of education
a. Primary school
b. Secondary school
c. High school
d. University

4. Marital status
a. Single
b. Married 
c. Separated/divorced

d. Widow

e. Unknown

5. Smoking habit
a. Never
b. Occasionally
c. Frequently

6. Drinking habit
a. Never
b. Occasionally
c. Frequently

7. Parity
a. One child
b. Two children
c. Three or more

8. Knowledge of one's health
a. Poor
b. Medium
c. Good

9. Hormonal pill contraception use
a. Never
b. Occasional
c. Frequent

10. Condom use during vaginal sex with current partner
a. Never
b. Sometimes
c. Always

11. Condom use during vaginal sex with clients
a. Never
b. Sometimes
c. Always

12. Have you ever had a Pap smear?
a. Never
b. Sometimes
c. Always

13. Have you ever had an STI?
a. Yes
b. No

14. Do you know that viruses may cause some types of cancer?
a. Yes
b. No

15. Have you heard about HIV infection?
a. Yes
b. No

16. Have you heard about HPV infection?
a. Yes
b. No

17. Have you heard about the HPV vaccine?
a. Yes
b. No

18. Would you like to receive an HPV vaccine?
a. Yes
b. No

19. Who informs you about how you should care for your health?
a. NGOs
b. Friends
c. Internet or media

The first part included 7 questions involving the participants' sociodemographic background. Demographic questions included age, country, education, marital status, smoking habit, drinking habit, and parity. The second part included 4 items examining the subjects' knowledge of their own health, hormonal contraception, condoms, and Pap smears. In this part, the subjects answered whether they had ever had a Pap smear. In the third part, knowledge about HPV was assessed using a 3-item scale. In addition, this part included questions about previous STIs, as well as their connection with cervical cancer, and transmission. The fourth part of the survey examined both knowledge about HIV, HPV, and attitudes toward HPV vaccination. Finally, the participants were asked about their source of health information

In addition, we obtained the participants' complete medical histories, including weight, height, and previous and current diseases.

\section{Statistical Analysis}

The proportions of HPV and HIV awareness were determined by age and country. Multiple logistic regression analysis was used to study the relationship between HPV and HIV awareness and the explanatory variables, which were selected based on the current literature. Interactions between geographic origin and all variables entered in the regression were examined.

We used likelihood ratio tests and Wald tests to calculate the $p$-values. A multiple logistic regression model was built incorporating all variables from univariate analyses. A backward approach was chosen, and the variables included in the final regression models were those that maximized the likelihood values. We tested linear relationships between ordered categorical variables and the log odds of knowledge of HIV and HPV infection. The relationships were assessed by comparing the models, assuming either the absence or presence of a linear relationship between the variable and the log odds of knowledge of HIV and HPV infection through likelihood ratio tests. 
Analyses were conducted using PASW Statistics 18 by estimating odds ratios (OR) and their corresponding 95\% confidence intervals (CI). Multivariate analyses for HIV and HPV were initially performed separately for the different variables.

\section{RESULTS}

The survey was conducted between July 2010 and July 2011. One hundred fifty-three women (aged 18-49 years) agreed to participate in the survey. Their mean (SD) age was 28.2 (7.41) years (range, 18-49 years); 10\% of participants were younger than 20 years, and $75 \%$ were younger than 35 (Fig. 1). They were predominantly from Latin America $(35.1 \%)$, followed by Spain $(23.08 \%)$, Eastern Europe (24.52\%), and Africa (17.31\%). Regarding education, $57.5 \%$ of the participants were illiterate (although they could read and write as different NGOs teach them), 33.5\% had graduated from primary school, $7.8 \%$ had finished high school, and 1.2\% (only women from Eastern Europe) had a university degree or more (Table 1). Regarding smoking status, $49.1 \%$ and $29.3 \%$ of the women were occasional and habitual smokers, respectively. Most (53.6\%) had at least 1 child, and $14.9 \%$ had 2 or more.

The majority of participants (79\%) were occasional drinkers. The women were asked whether they had ever had a Pap smear; $52.7 \%(\mathrm{n}=88)$ indicated that they had never had one. Regarding self-rated health, $49.1 \%$ reported poor knowledge, $47.3 \%$ moderate, and $3.6 \%$ had no idea.

Over $90 \%$ of the women reported penetrative vaginal sex with either regular partners or clients. Although condom use was high with clients - only $11.3 \%$ did not use condoms with clients $-70 \%$ did not use them with regular partners (Fig. 2).

Condom use with clients was lower among women from other countries than women from Spain $(\mathrm{p}=0,001$, OR,

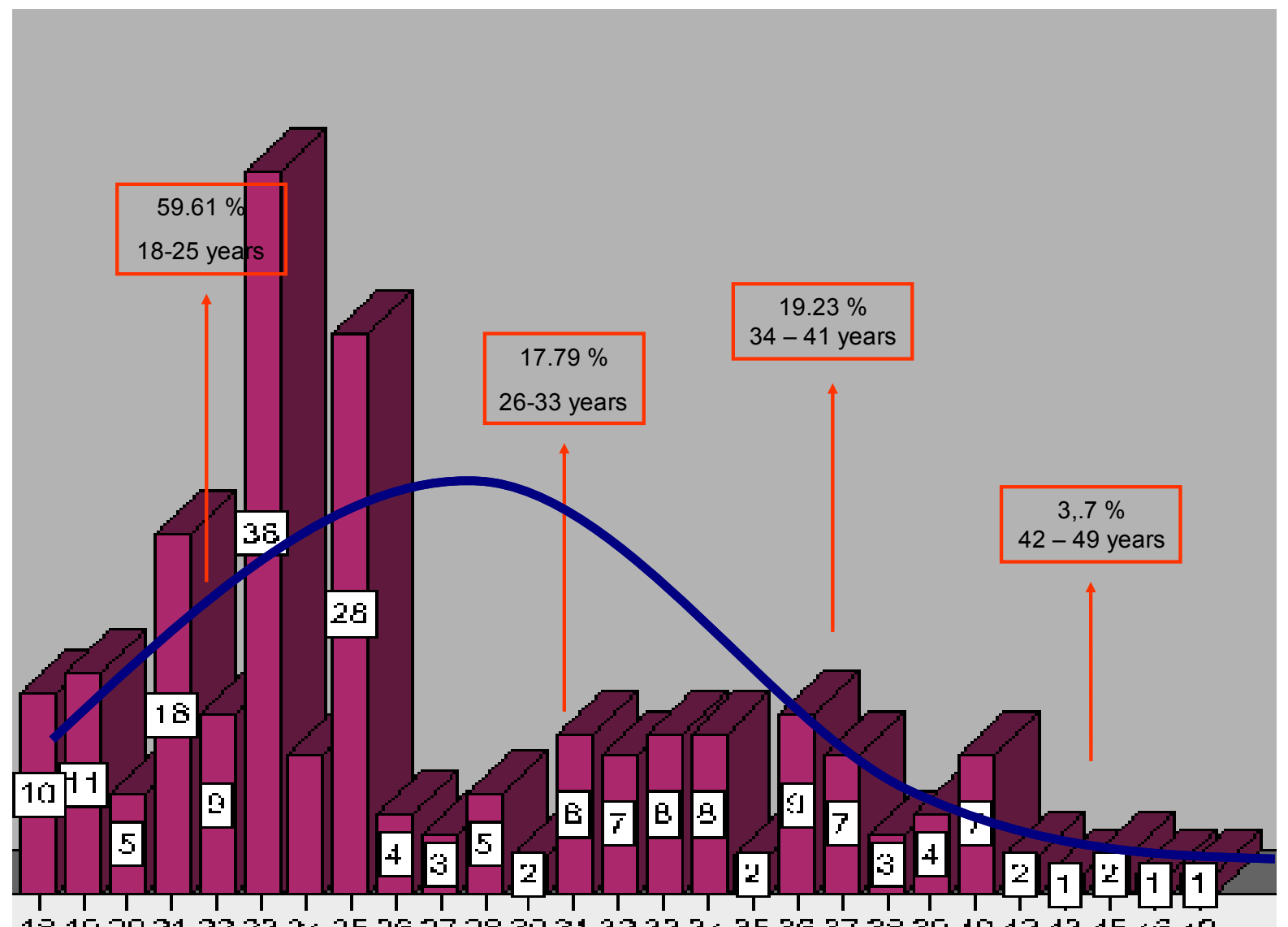

$1819202122232<25262728303132333<353637383940424345 \angle 649$

Fig. (1). Their mean (SD) age was 28.2 (7.41) years (range, 18-49 years).

Table 1. The largest group of female sex worker participating in our study consists of immigrant women.

\begin{tabular}{|c|c|c|c|c|c|}
\hline AGE & $\mathbf{1 8 - 2 5}$ & $\mathbf{2 6 - 3 3}$ & $\mathbf{3 4 - 4 1}$ & $\mathbf{4 2 - 4 9}$ & Total \\
\hline \hline Spain & $16(7,69 \%)$ & $9(4,33 \%)$ & $16(7,69 \%)$ & $7(3,37 \%)$ & $0(0 \%)$ \\
\hline Eastern Europe & $38(18,27 \%)$ & $8(3,85 \%)$ & $5(2,40 \%)$ & $0(0 \%)$ & $\mathbf{2 3 , 0 8 \%}$ \\
\hline Latin America & $48(23,08 \%)$ & $12(5,77 \%)$ & $13(6,25 \%)$ & $0(0 \%)$ & $\mathbf{3 5 , 1 \%}$ \\
\hline Africa & $22(10,58 \%)$ & $8(3,85 \%)$ & $6(2,88 \%)$ & $3,37 \%$ & $\mathbf{1 7 , 3 1 \%}$ \\
\hline Total & $59,61 \%$ & $17,79 \%$ & $19,23 \%$ & $\mathbf{1 0 0} \%$ \\
\hline
\end{tabular}




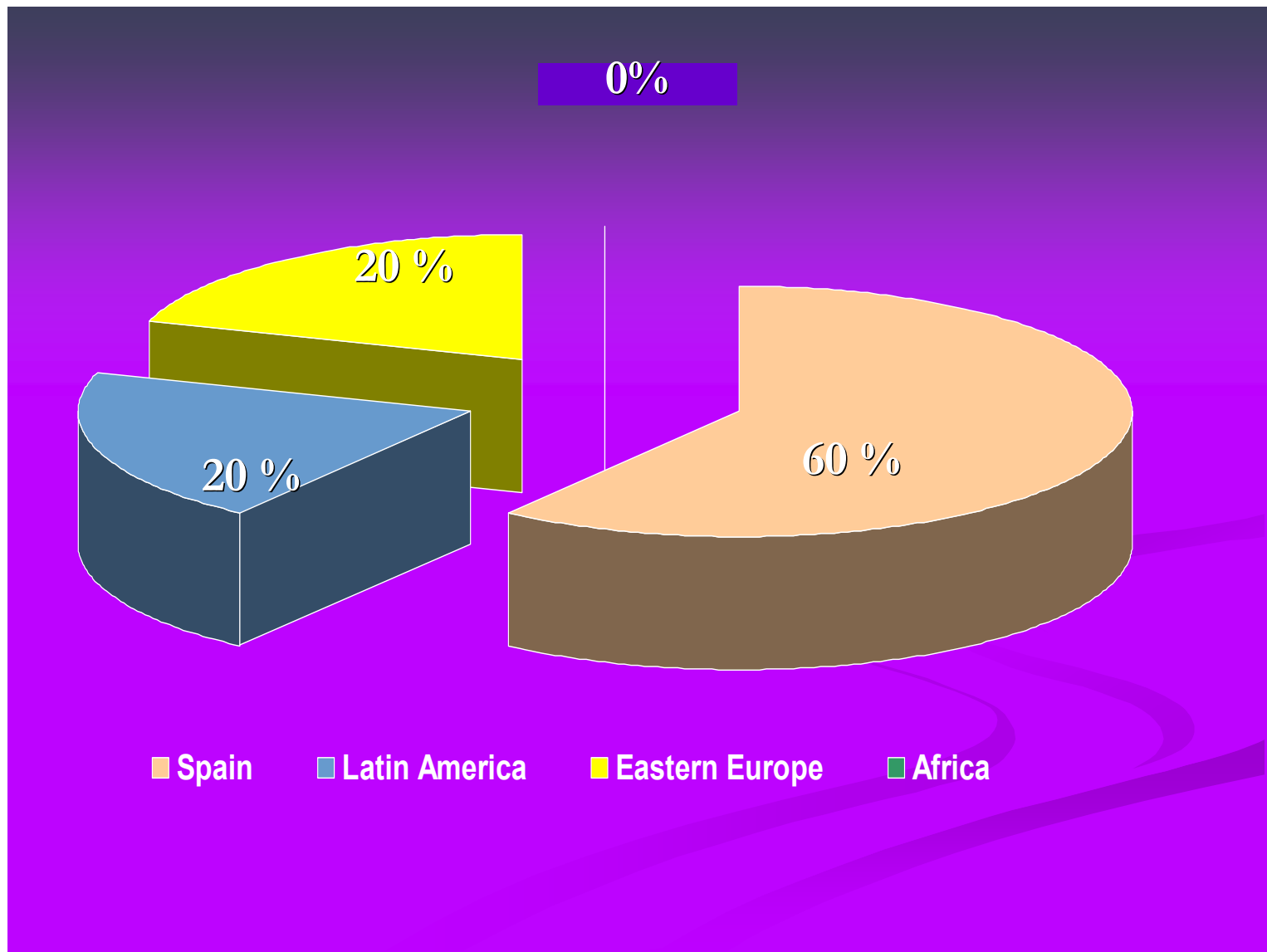

Fig. (2). The male condom use both vaginal intercourse with clients and in the private sphere is highly significant in relation to country of origin.

0.136; 95\% CI, 0.049-0.381), whereas condom use with regular partners was more frequent $(\mathrm{p}=0,005$, OR, 0.36 95\% CI, 0.049-0.381) among Spanish women. Condom breakeage with clients in the month before the survey were high ( 45\%). Eastern European women had a risk that was 2.941-fold higher than that of Spaniards of not knowing about HPV vaccines.

There was no relationship between country or age and knowledge about HIV (Fig. 3). Eastern European women knew less (OR, 2.941; 95\% CI, 1.071-8.074). Regarding vaccines, women who had been tested for HIV and had a history of Pap smears knew more HPV. These findings are useful for future HPV vaccination campaigns and may be particularly useful for developing intervention programs for individuals with the largest deficits in HPV knowledge.

Participants who always used condoms with their clients knew significantly more about vaccines (95\% CI, 0.115 $0.790)$ than those who never used condoms with clients (95\% CI, 0.036-0.623) ( $p=0.009)$. In addition, the participants with a history of any STI knew significantly more about the existence of HPV vaccines $(80 \% ; 95 \% \mathrm{CI}$, $0.103-0.473)(p=0.000)$. In all, $10.3 \%$ of participants were HIV-positive. Information on the HIV status of current partners was available for $100 \%$ of the women who were HIV-positive; $89.4 \%$ of their current partners were HIVpositive as well. There were no differences in HIV prevalence between women who had engaged in sex work for $1-3$ years $(31.1 \%), 3-5$ years $(44.9 \%)$, and $>5$ years $(23.4 \%)$.

When the participants were asked if they knew viruses might cause some types of cancer, $42.9 \%$ of respondents answered correctly, whereas $56.5 \%$ did not know. Most $(88 \%)$ of the women responded correctly to the question about HIV infection and their consequences. Moreover, $41 \%$ of the respondents had heard of a vaccine for cervical cancer. Most had heard about caring for themselves from NGOs $(68.3 \%)$ and the internet or mass media (13.8\%) (Fig. 3). The majority $(56 \%)$ of respondents had never heard of HPV. Among the women who had never heard of HPV (46.1\%), $99 \%$ knew about vaccines and had a positive attitude toward them.

Although $82.7 \%$ of the participants were willing to be vaccinated, only $3 \%$ of them were vaccinated at that time. On the other hand, $21 \%$ of the subjects unwilling to be vaccinated had doubts about the new vaccine or did not have enough money. For most of the women $(65.4 \%)$, recommendations from NGO volunteers were the major influences for deciding to be vaccinated.

\section{DISCUSSION}

The main goal of this study was to guide future HIV prevention efforts in this high-risk population in Northwestern Spain by assessing the current knowledge of FSW on HIV, determining the role of injection drug use in the epidemic, and determining other risk factors for HIV 


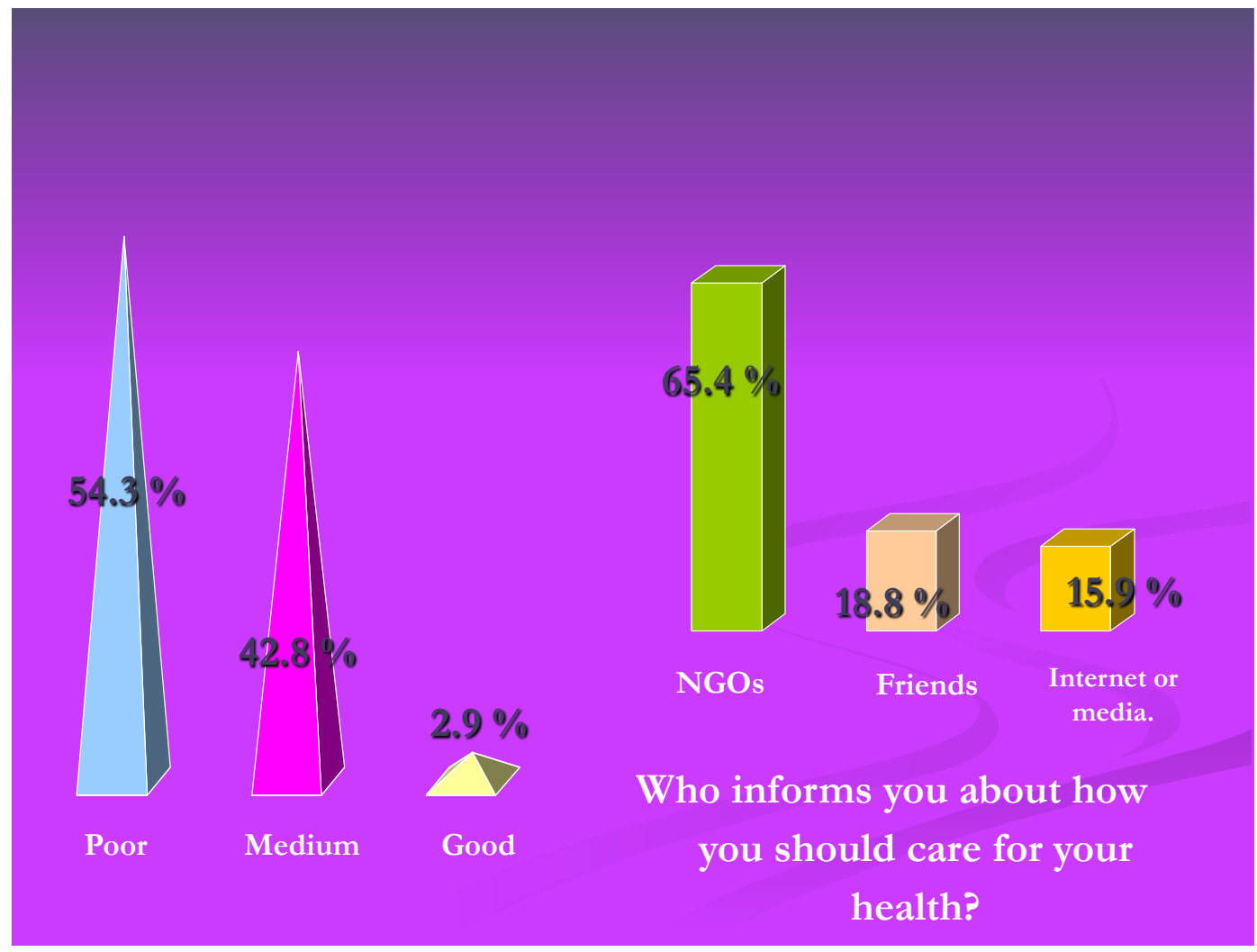

Fig. (3). Knowledge of one's health.

infection. The most frequent transmission route in cases of AIDS in Galicia, Spain, is associated with injected drugs, which corresponded to $43.4 \%$ of all cases in 2008 [16]. On the other hand, heterosexual transmission is the second most frequent route, corresponding to $30.1 \%$ (48\% in women) of cases in 2008. Nonetheless, recent data from other Spanish communities should be taken into account. It is also possible that AIDS incidence will increase over the coming years in this group. The most common geographic area from which the immigrants originate is Latin America (48\% of cases; 33 men and 28 women) followed by Europe (32\%), and subSaharan Africa (15\%). The most common mode of transmission of HIV, in this group, is heterosexual transmission, which comprises $44 \%$ of cases (56 of 127), followed by shared syringe use among injection drug users, comprising $29 \%$ of cases. In Europeans, the most common mode of transmission is unsafe sexual behavior, involving 27 of 41 cases $(66 \%)$ in total; homo- and bisexuals represent $17 \%$ of these cases [16].

When asked, "Have you ever heard of HIV?" Spaniards and Africans knew significantly more than Eastern Europeans and Latin Americans did (Fig. 2).

On the other hand, the prevalence of HPV in the female lower genital tract in Northwestern Spain is still high; infection was prevalent in young women, indicating a decrease in infection in middle age. However, postmenopausal women exhibited higher infection rates. The HPV-16 serotype was the most prevalent $(27 \%)$, followed by HPV-31 (11\%), HPV-52 (7\%), HPV-56 (5\%), and HPV-58 $(2 \%)$. Multiple infections were present in $38 \%$ of the participants and decreased with age. The HPV-16 and HPV31 serotypes exhibit similar behavior in both single and multiple infections $[17,18]$. This study presents the first data on the incidence of HPV in FSW. With the use of vaccines against HPV - especially the tetravalent vaccine - there will be a lower rate of abnormal cytological results in vaccinated women in the short- or medium-term; this is based on the vaccine's expected preventive potential, which may range $50-70 \%$. Furthermore, with the use of the vaccine in combination with present screening programs, we could reduce the annual number of cases of cervical cancer and other associated pathologies in Spain by $92 \%$. We obtained these data in a study in a normal population [17].

In order to assess the public's understanding of HPV vaccination and testing, it is important to evaluate knowledge of HPV and its relationship with genital cancer. In the current population-based study that included 153 women who were 18-45 years old, we found that, overall, $46 \%$ reported having heard of HPV. This proportion ranged from $30.4 \%$ in Latin Americans to $19.6 \%$ in Africans. The results of this study corroborate the findings of other studies in that many women in the general population still do not know about HPV. Moreover, even fewer FSW know about HPV. Less is known about the existence of HPV than of HIV.

In a major Catalonian study of over 350 immigrant FSW from Eastern Europe, Latin America, and Africa, Folch et al. [19] found that younger age and unprotected sex were associated with gonorrhea and chlamydia. They note that over $70 \%$ of FSW in Spain now are immigrants and that precarious legal (70\% are illegal immigrants), working, and family situations, as well as poor access to social services may make these women more vulnerable to STIs. However, they found that the prevalence of STIs among migrant FSW 
in Spain is lower than that in other European countries. Furthermore, they also found that the rates of chlamydia and gonorrhea are consistent with those in other sexually active young people in Catalonia. None of the women in this study were injection drug users, which may also account for the low prevalence of health problems and HIV, although there was no direct comparison with non-immigrant FSW. We found a very low rate of HIV among the FSW, and STIs data did not differ greatly from the normal population.

FSW from Eastern Europe (who were also the youngest) knew the most about HPV, whereas those from Africa knew the least. Although these differences were of borderline statistical significance due to the small number of women in some groups, they were maintained in age-adjusted analyses. Therefore, the country of origin must reflect differences in either past or current sexual behaviors. Unfortunately, no detailed data were available for all women [14].

The fact that no differences were observed for acute STIs by geographic origin suggests that past exposure to HPV may be a determinant of current HPV epidemiology.

There is strong evidence that FSW have unsafe sex, as highlighted by the high prevalence of terminated pregnancies (78.5\%) and other STIs (50.3\%).

Furthermore, unsafe sex took place more frequently with regular partners than clients [20].

The prevalence of STIs was neither related to age nor in the patients from different geographic areas.

Multivariate analysis revealed that knowledge about the association between HIV and its consequences and the relationship between education and condom use with partners were strongly associated. Related to the knowledge of the association between HPV and cervical cancer, knowledge of the routes of HPV transmission and awareness about vaccines among the FSW was strongly associated with education, knowledge of one's health, parity, and condom use with clients [21].

Moreover, previous knowledge about HPV and the HPV vaccine; personal beliefs about vaccination; many sexual partners; perceived support of providers, partners, and parents; endorsement of universal HPV vaccination; low vaccine cost; and vaccine safety were all significantly associated with the acceptance of HPV vaccination [22-24].

Alcohol and drug use are frequently associated with sex work either because the nature of the work leads to selfmedication, or because sex work is a relatively easy way to obtain money to finance a drug habit. However, the specific context of sex work may also require sex workers to consume alcohol. In a classic study, Fernández-Esquer [25] studied cantineras, Latinas working in lower-class bars (cantinas) in North and Central America who earn a sales commission from every beer they get clients to buy for them.

In Northwestern Spain, drinking is virtually obligatory for FSW and is paid for by men who expect sexual favors in exchange. A female sex worker may consume up to 8 beers during a "normal" working day. In such cases, alcohol use is an integral part of the work and is not a precursor or consequence of the work; however, it is still a female sex worker issue. Similar concerns are warranted for other sex workers in bars, where they also work as waitresses and are expected to encourage alcohol consumption by patrons and potential clients. The health consequences of high and onsistent alcohol consumption - apart from the immediate safety issues of unsafe sex and poor judgment in the selection of clients who may be abusive - may include long-term liver disease, heart disease, obesity, and depression, among others [26, 27]. In the present study, $79 \%$ of the participants were casual drinkers, $48 \%$ presented liver disease, and $2.4 \%$ had heart disease; height ranged 1.50-1.68 $\mathrm{m}$ and weight was $57-75 \mathrm{~kg}$ with an average BMI of $30 \%$ [28].

Finally, the time spent in commercial sex work was significantly associated with HIV knowledge. However, information on the time spent in commercial sex work is often missing, and past history of STIs is also susceptible to recall bias. It is likely that a certain random misclassification may have taken place, which could explain the lack of an observed association [29].

\section{CONCLUSION}

In conclusion, this study confirms that more education about HIV, HPV, and HPV vaccination is urgently needed for women. This is the first study to assess what FSW in Northwestern Spain know about HIV, HPV, and HPV vaccines; it provides useful information for further research and policy makers. Social care professionals should advise FSW about care and vaccines. It is important to convey preventative messages to the personal sphere of FSW (i.e., their private partners and clients) and to facilitate the access of FSW to the health system. Since women may feel uncomfortable talking about STIs, it is important to be mindful of this when explaining HIV, HPV, HPV vaccination, and genital cancer. The important findings of the present study highlight the need for effective education for practitioners, social workers, and NGO volunteers. It is also important to remember that the government should support such programs, including assistance and vaccines, for this low-income group.

\section{CONFLICT OF INTEREST}

The authors confirm that this article content has no conflict of interest.

\section{ACKNOWLEDGEMENTS}

Declared none.

\section{REFERENCES}

[1] Seib C, Fischer J, Najman JM. The health of female sex workers from three industry sectors in Queensland, Australia. Soc Sci Med 2009; 68 (3): 473-8.

[2] Zhang C, Li X, Hong Y, Chen Y, Liu W, Zhou Y. Partner violence and HIV risk among female sex workers in China. AIDS Behav 2011; (16)4: 1020-30.

[3] Hong Y, Li X. Behavioral studies of female sex workers in China: a literature review and recommendation for future research. AIDS Behav 2008;12(4): 623-36.

[4] Groneberg DA, Molliné M, Kusma B. Sex work during the world cup in Germany. Lancet 2006; 368: 840-1.

[5] Alexander P. Sex work and health: A question of safety in the workplace. J Am Med Women's Assoc 1998; 53: 77-82.

[6] Montano SM, Hsieh EJ, Calderón M, Ton TG, Quijano E, Solari V, Zunt JR. Human papillomavirus infection in female sex workers in Lima, Peru. Sex Transm Infect 2011; 87 (1): 81-2. 
[7] Bautista CT, Pando MA, Reynaga E, et al. Sexual practices, drug use behaviors, and prevalence of HIV, syphilis, hepatitis B and C, and HTLV-1/2 in immigrant and non-immigrant female sex workers in Argentina. J Immigr Minor Health 2009; 11: 99-104.

[8] Plumridge L, Abel G. A "segmented" sex industry in New Zealand: Sexual and personal safety of female sex workers. Aust NZ J Public Health 2001; 25: 78-83.

[9] Sanders T. A continuum of risk? The management of health, physical and emotional risks by female sex workers. Soc Health Ill 2004; 26: 557-74.

[10] Farley M, Cotton A, Lynne J, et al. Prostitution and trafficking in nine countries: an update on violence and posttraumatic stress disorder. J Trauma Pract 2003; 3: 3374 .

[11] Langanke H, Ross MW. Web-based forums for clients of female sex workers: development of a German internet approach to HIV/STD-related sexual safety. Int J STD AIDS 2009; 20 (1): 4-8.

[12] Hong Y, Li X, Fang X, Lin X, Zhang C. Internet use among female sex workers in China: Implications for HIV/STI prevention. AIDS Behav 2011; 15(2): 273-82

[13] Nguyen NT, Nguyen HT, Trinh HQ, Mills SJ, Detels R. Clients of female sex workers as a bridging population in Vietnam. AIDS Behav 2009; 13. (5): 881-91.

[14] Barrington C, Latkin C, Sweat MD, Moreno L, Ellen J, Kerrigan D. Talking the talk, walking the walk: social network norms, communication patterns, and condom use among the male partners of female sex workers in La Romana, Dominican Republic. Soc Sci Med 2009; 68 (11): 2037-44.

[15] Yang C, Latkin C, Luan R, Nelson K. Peer norms and consistent condom use with female sex workers among male clients in Sichuan province, China. Soc Sci Med 2010; 71 (4): 832-9.

[16] Rodriguez-Cerdeira C, Cruces MJ, Taboada JA. A quarter century with AIDS. Open AIDS J 2011; 1: 1-8

[17] Rodríguez-Cerdeira MC, Guerra-Tapia A, Alcantara CR, Escalas J. Human Papilloma Virus (HPV) and genital cancer. Open Dermatol J 2009; 3: 111-22.

[18] Rodríguez-Cerdeira C, Chillón R, Díez-Moreno S, Guerra-Tapia A. Prevalence and genotypic identification of human papillomavirus infection in a population from northwestern Spain. Open Dermatol J. 2009; 3: 18-21.

[19] Folch C, Esteve A, Sanclemente C et al. Prevalence of HIV, Chlamydia trachomatis, and Neisseria gonorrheae and risk factors for sexually transmitted infections among immigrant female sex workers in Catalonia, Spain. Sex Transm Dis 2008; 35: 178-83.

[20] Hoffman L, Nguyen HT, Kershaw TS, Niccolai LM. Dangerous subtlety: relationship-related determinants of consistency of condom use among female sex workers and their regular, noncommercial partners in Haiphong, Vietnam AIDS Behav. 2011;15: 1372-80.

[21] Sanders T. Protecting the health and safety of female sex workers: the responsibility of all. BJOG 2007; 114 (7): 791-3.

[22] Gonik B. Strategies for fostering HPV vaccine acceptance. Infect Dis Obstet Gynecol 2006; 36797: 1-4.

[23] Kahn JA, Rosenthal SL, Hamann T, Bernstein DI. Attitudes about human papillomavirus vaccine in young women. Int J STD AIDS 2003; 14: 300-6.

[24] Boehner CW, Howe SR, Bernstein DI, Rosenthal SL. Viral sexually transmitted disease vaccine acceptability among college students. Sex Transm Dis 2003; 30: 774-8.

[25] Fernández-Esquer ME. Drinking for wages: Obligatory alcohol abuse among Cantineras. J Stud Alcohol. 2003; 64: 160-6.

[26] Parks KA, Hsieh YP, Lorraine CR, Levonyan-Radloff K. Daily assessment of alcohol consumption and condom use with known and casual partners among young female bar drinkers. AIDS Behav. 2011; 15(7): 1332-41.

[27] Samet JH, Pace CA, Cheng DM, et al. Alcohol use and sex risk behaviors among hiv-infected female sex workers and HIVinfected male clients of female sex workers in India. AIDS Behav. 2010; 14(Suppl 1): S74-83.

[28] Kershaw TS, Arnold A, Lewis JB, Magriples U, Ickovics JR. The skinny on sexual risk: the effects of bmi on sti incidence and risk. AIDS Behav. 2011; 15 (7): 1527-38.

[29] Belza MJ, Clavo P, Ballesteros J, et al. Social and work conditions, risk behavior and prevalence of sexually transmitted diseases among female immigrant prostitutes in Madrid (Spain) Gac Sanit. 2004; 18 (3): 177-83.

(C) Rodríguez-Cerdeira et al.; Licensee Bentham Open.

This is an open access article licensed under the terms of the Creative Commons Attribution Non-Commercial License (http://creativecommons.org/licenses/by-nc/3.0/) which permits unrestricted, non-commercial use, distribution and reproduction in any medium, provided the work is properly cited. 